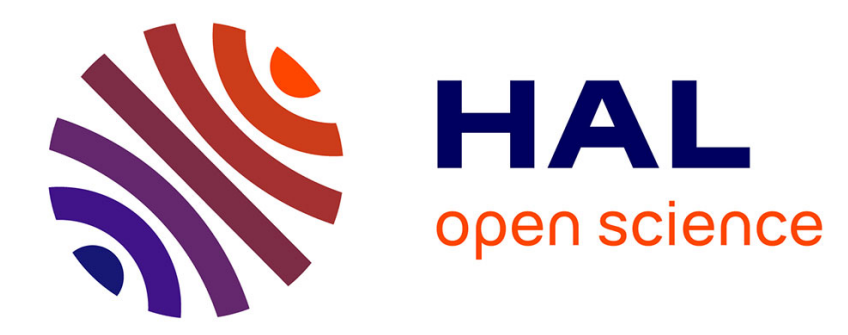

\title{
Entropy and maximizing measures of generic continuous functions
}

\author{
Julien Brémont
}

\section{To cite this version:}

Julien Brémont. Entropy and maximizing measures of generic continuous functions. CRAS, 2008, I (346), pp.199-201. hal-00794126

\section{HAL Id: hal-00794126 \\ https://hal.science/hal-00794126}

Submitted on 25 Feb 2013

HAL is a multi-disciplinary open access archive for the deposit and dissemination of scientific research documents, whether they are published or not. The documents may come from teaching and research institutions in France or abroad, or from public or private research centers.
L'archive ouverte pluridisciplinaire HAL, est destinée au dépôt et à la diffusion de documents scientifiques de niveau recherche, publiés ou non, émanant des établissements d'enseignement et de recherche français ou étrangers, des laboratoires publics ou privés. 


\title{
ENTROPY AND MAXIMIZING MEASURES OF GENERIC CONTINUOUS FUNCTIONS
}

\author{
Julien Brémont
}

Université Paris 12, novembre 2007

\begin{abstract}
In the natural context of ergodic optimization, we provide a short proof of the assertion that the maximizing measure of a generic continuous function has zero entropy.
\end{abstract}

\begin{abstract}
Dans le cadre usuel de l'étude des mesures maximisantes, nous donnons une preuve courte du fait que la mesure maximisante d'une fonction continue générique est d'entropie nulle.
\end{abstract}

\section{Introduction}

Let $(X, T)$ be a topological dynamical system, where $X$ is a compact metric space with a continuous transformation $T: X \longmapsto X$. Introduce the set $\mathcal{M}_{T}$ of Borel $T$-invariant probability measures on $X$, endowed with the compact and metrizable weak-* topology. We assume that measures supported by a periodic orbit are dense in $\mathcal{M}_{T}$ and that the map $\mu \longmapsto h(\mu)$ is upper-semi-continuous (usc) on $\mathcal{M}_{T}$. These assumptions are for instance verified if $(X, T)$ satisfies expansiveness and specification (cf Denker-Grillenberger-Sigmund [5]).

Fixing a continuous $f: X \rightarrow \mathbb{R}$, "ergodic optimization" (see Jenkinson [6] and references therein) is concerned with the following variational problem :

$$
\beta(f)=\sup \left\{\mu(f) \mid \mu \in \mathcal{M}_{T}\right\} \text { and } \mathcal{M a x}(f)=\left\{\mu \in \mathcal{M}_{T} \mid \beta(f)=\mu(f)\right\},
$$

where $\mu(f)$ is for $\int f d \mu$. The aim is to describe the set Max $(f)$ of maximizing measures for $f$, which is always a non-empty compact and convex subset of $\mathcal{M}_{T}$. Notice also that any measure in the ergodic decomposition of a maximizing measure is a maximizing measure. We consider here genericity results in functional spaces. Recall that a set is residual if it contains a dense $G_{\delta^{-}}$-set. A property defining a residual set is generic. An element in a residual set is declared generic.

The regularity of $f$ plays a crucial role. In a Hölder or Lipschitz functional space, the ConzeGuivarc'h-Mañé lemma (see [6] for instance) gives a characterization of the maximizing measures via their support. The analysis is fairly delicate and difficult conjectures about periodic measures remain open (cf [6], [3] and references therein). The analysis of the case of the space $C(X)$ of realvalued continuous functions on $X$ (endowed with the supremum norm) is completely different. The Conze-Guivarc'h-Mañé lemma is not valid any more, but duality arguments are available. Bousch and Jenkinson $[1,2]$ showed that for a generic $f$ in $C(X)$ the situation is somehow pathological.

Theorem 1.1 (Bousch-Jenkinson)

A generic function in $C(X)$ has a unique maximizing measure and it has full support.

AMS 2000 subject classifications : 37D99.

Key words and phrases : generic continuous function, maximizing measure, entropy. 
In a recent article on a closely related problem, Jenkinson and Morris [7] considered the entropy of "Lyapunov maximizing measures" for $C^{1}$-expanding maps of the Circle. Certainly, their method allows to complete the picture in the following way, in some sense restricting the "pathology" :

Theorem 1.2 (Jenkinson-Morris)

The maximizing measure of a generic function in $C(X)$ has zero entropy.

The purpose of this note is to give a short and rather elegant proof of the latter result. Let us also mention that in the particular case of a symbolic setup (such as the shift $T$ on some product space $X=\{0, \cdots, m-1\}^{\mathbb{Z}}$ ), theorem (1.2) can be proved elementarily using the density in $C(X)$ of locally constant functions, cf Conze-Guivarc'h [4].

\section{Proof of theorem 1.2}

Define a non-negative map $\varphi: f \longmapsto \sup _{\mu \in \mathcal{M} a x(f)} h(\mu)$ on $C(X)$. Let us check that it is usc. Indeed, since $\mu \longmapsto h(\mu)$ is usc and $\mathcal{M a x}(f)$ is compact, $\varphi(f)=h\left(\mu_{f}\right)$ for some $\mu_{f} \in \operatorname{Max}(f)$. If now $f_{n} \rightarrow f$, then up to extraction $\mu_{f_{n}}$ weakly converges to some $\mu$ in $\mathcal{M} a x(f)$ and thus $\varphi(f) \geq h(\mu) \geq \lim \sup h\left(\mu_{f_{n}}\right)$. This proves the assertion.

As a result, $\varphi$ is continuous on a residual set $R$. We will show that $\varphi$ in restriction to $R$ is equal to zero. This latter fact will be a corollary from the following claim, of independent interest.

Proposition 2.1 On a dense set $D$ in $C(X)$, the maximizing measure is unique and supported by a periodic orbit.

Assuming this result, let $f \in R$ and $f_{n} \rightarrow f$ with $f_{n}$ in $D$. Since $\varphi\left(f_{n}\right)=0$ and $\varphi$ is continuous at $f$, we get $\varphi(f)=0$. Thus $\varphi_{\mid R}$ is zero, as announced. This gives theorem (1.2).

To prove the latter proposition, first notice that it is enough to show that densely in $C(X)$ there is a periodic maximizing measure. Indeed, if $g$ has a maximizing measure $\mu$ supported by some periodic orbit $\operatorname{Orb}\left(x_{0}\right)$, introduce for $\eta_{0}>0$ the map $\eta(x)=-\eta_{0} \operatorname{dist}\left(x, \operatorname{Orb}\left(x_{0}\right)\right), \forall x \in X$. Then for $\nu \in \mathcal{M}_{T}$, one has $\nu(g+\eta)=\nu(g)+\nu(\eta)$ and $\nu(g) \leq \beta(g)$ and $\nu(\eta) \leq 0$, with both equalities simultaneously if and only if $\nu=\mu$. We therefore obtain $\mathcal{M a x}(g+\eta)=\{\mu\}$ and this gives the result since $\|\eta\|_{\infty} \rightarrow 0$ as $\eta_{0} \rightarrow 0$.

To conclude, take any $f$ and a measure $\mu \in \mathcal{M}_{T}$ supported by a periodic orbit with small $\beta(f)-\mu(f)$. By the next proposition, $f$ can be perturbed into $g$ with a maximizing measure $\nu$ such that $\mu$ and $\nu$ are not mutually singular (taking $\varepsilon=1 / 2$ in the statement of the proposition). As $\mu$ is ergodic, it appears in the ergodic decomposition of $\nu$ and thus $\mu \in \operatorname{Max}(g)$.

The next proposition comes from the classical proof of the Bishop-Phelps theorem. It is adapted from a preliminary version of Pollicott and Sharp [8].

Proposition 2.2 Let $f \in C(X)$ and $\mu \in \mathcal{M}_{T}$. Write $\beta(f)-\mu(f)=\varepsilon \delta$, with $\varepsilon \geq 0, \delta \geq 0$. Then there exist $g \in C(X)$ and $\nu \in \mathcal{M a x}(g)$ such that $\|f-g\|_{\infty} \leq \delta$ and $\|\mu-\nu\|_{C(X)} \leq \varepsilon$.

Proof of the proposition: From homogeneity and the fact that $\operatorname{Max}(g)=\operatorname{Max}(\lambda g)$ for $\lambda>0$, it is enough to suppose that $\delta=1$. Clearly we can also assume that $\varepsilon>0$. Define $\Phi(u)=\beta(u)-\mu(u)$ on $C(X)$ and let, for $v \in C(X)$ :

$$
A(v)=\left\{u \in C(X) \mid \Phi(u) \leq \Phi(v)-\varepsilon\|v-u\|_{\infty}\right\} .
$$

By the triangular inequality, observe that $A(u) \subset A(v)$ if $u \in A(v)$. Let now $f_{0}=f$, with $\Phi\left(f_{0}\right)=\varepsilon$, and for $n \geq 0$, choose $f_{n+1} \in A\left(f_{n}\right)$ such that $\Phi\left(f_{n+1}\right) \leq 2^{-n-1} \varepsilon+\inf \left\{\Phi(u) \mid u \in A\left(f_{n}\right)\right\}$. Then $\left(A\left(f_{n}\right)\right)$ is decreasing and one has for $n \geq 0$ and any $u \in A\left(f_{n}\right)$ :

$$
\Phi\left(f_{n}\right)-\varepsilon 2^{-n} \leq \Phi(u) \leq \Phi\left(f_{n}\right)-\varepsilon\left\|f_{n}-u\right\|_{\infty} .
$$


As a result $\left\|f_{n}-u\right\|_{\infty} \leq 2^{-n}$. Thus $\left(f_{n}\right)$ is a Cauchy sequence converging to some $g$ and $\operatorname{diam}\left(A\left(f_{n}\right)\right) \leq 2^{-n+1}$. Therefore $\|f-g\|_{\infty} \leq 1$ and $A(g)=\{g\}$.

By this last property of $g$, the open convex set $\left\{(u, y) \in C(X) \times \mathbb{R} \mid y<\Phi(g)-\varepsilon\|g-u\|_{\infty}\right\}$ and the convex set $\{(u, y) \in C(X) \times \mathbb{R} \mid y \geq \Phi(u)\}$ are disjoint. From the Hahn-Banach separation theorem (cf Ruelle [9], Appendix A.3.3 (a)), there is a linear form $L(u, y)=y-\tilde{\mu}(u)$, with a signed Borel measure $\tilde{\mu}$, and $t \in \mathbb{R}$ such that for all $u \in C(X)$ :

$$
\Phi(g)-\varepsilon\|g-u\|_{\infty}-\tilde{\mu}(u) \leq t \leq \Phi(u)-\tilde{\mu}(u) .
$$

Taking $u=g$ gives $t=\Phi(g)-\tilde{\mu}(g)$. Thus for all $u \in C(X)$, we have $\Phi(g)-\tilde{\mu}(g-u) \leq \Phi(u)$ and $\tilde{\mu}(g-u) \leq \varepsilon\|g-u\|_{\infty}$, which can be rewritten as $\beta(g)+(\mu+\tilde{\mu})(u) \leq \beta(g+u)$ and $|\tilde{\mu}(u)| \leq \varepsilon\|u\|_{\infty}$.

Consequently and by definition, $\nu=\mu+\tilde{\mu}$ is a tangent functional for $\beta$ at $g$ (cf Ruelle [9], Appendix A.3.6). As detailed in the next lemma, it is thus a maximizing measure for $g$.

Lemma 2.3 Let $f \in C(X)$ and a signed Borel measure $\nu$ be such that $\beta(f)+\nu(g) \leq \beta(f+g)$, for all $g \in C(X)$. Then $\nu$ is an invariant probability measure and it belongs to Max $(f)$.

Proof of the lemma: Let $g \geq 0$ in $C(X)$. Since $\beta(f) \geq \beta(f-g)$, we get $\nu(g) \geq \beta(f)-\beta(f-g) \geq 0$. Thus $\nu$ is positive. Also for any real constant $a$, we have $\beta(f+a)=\beta(f)+a$, giving $\nu(a) \leq a$. Therefore $\nu(1)=1$ and $\nu$ is a probability measure.

Let $g \in C(X)$. Since $\beta(f+g-g \circ T)=\beta(f)$, we have $\nu(g-g \circ T) \leq 0$. Taking $-g$, we get equality. Thus $\nu$ is $T$-invariant. Next, as $\beta(0)=0$, when taking $g=-f$ we obtain $\beta(f)-\nu(f) \leq 0$. This shows that $\nu \in \operatorname{Max}(f)$ and concludes the proof of the lemma.

\section{References}

[1] Bousch, T., La condition de Walters, Ann. Sci. ENS, 34 (2001), 287-311.

[2] Bousch, T. And Jenkinson, O., Cohomology classes of dynamically non-negative $C^{k}$ functions, Invent. Math. 148, 207-217 (2002).

[3] BrÉmont, J., Finite flowers and maximizing measures for generic Lipschitz functions on the Circle, Nonlinearity, 2006, Vol. 19, 813-828.

[4] Conze, J.-P. And Guivarc'H, Y., Croissance des sommes ergodiques et principe variationnel, Tech. report, Université de Rennes 1, 1990.

[5] Denker, M., Grillenberger, C. And Sigmund, K., Ergodic theory on compact spaces, Springer-Verlag, Berlin, 1976, Lecture Notes in Mathematics, Vol. 527.

[6] Jenkinson, O., Ergodic optimization. Discrete and Cont. Dyn. Systems, 15 (2006), 197-224.

[7] Jenkinson, O. And Morris, I., Lyapunov optimizing measures for $C^{1}$ expanding maps of the Circle. Preprint 2007.

[8] Pollicott, M. And Sharp, R., Livsic theorems, maximizing measures and the stable norm, Dyn. Sys. 19 (2004), 75-88.

[9] Ruelle, D., Thermodynamic formalism, second edition, Cambridge University press, Cambridge (2004).

Laboratoire d'Analyse et de Mathématiques Appliquées, Université Paris XiI, Faculté des Sciences et Technologies, 61 avenue du Général de Gaulle, 94010 Créteil Cedex, FranCE E-mail address : bremont@univ-paris12.fr 